\title{
Agitated Saline Contrast Echocardiogram In Cardio-Pulmonary Evaluation
}

\author{
Anish Hirachan ${ }^{1}$, Ranjit Sharma ${ }^{1}$, Prabesh Neupane ${ }^{1}$ \\ ${ }^{1}$ Department of Intervention Cardiology, Nepal Mediciti Hospital , Kathmandu, Nepal
}

Corresponding Author: Anish Hirachan

Department of Intervention Cardiology, Nepal Mediciti Hospital, Kathmandu, Nepal

Email: hirachananish@gmail.com

ORCDID NO-0000-0001-7714-7295

Cite this article as: Hirachan A., Sharma R., Neupane P., et al. Agitated Saline Contrast Echocardiogram In Cardio-Pulmonary Evaluation. Nepalese Heart Journal 2021; Vol 18(2), 53-55.

Submission date: $24^{\text {th }}$ May, 2021

Accepted date: $11^{\text {th }}$ October, 2021

\section{Abstract}

Agitated saline contrast echocardiogram (ASC) is a very useful technique to detect various intracardiac and extra cardiac shunts in daily cardiology practice. Conventional 2D and color echocardiogram may not be well effective in ruling out various intracardiac shunts especially with patients having poor echo window. The introduction of agitated saline with bubbles formed during the study can help delineate different right to left shunt physiology commonly like patent foramen ovale (PFO) which is often sought for in evaluation of cases with young stroke . Various other etiologies like atrial septal defects, atrial septal aneurysm, large right to left shunts with eisenmengerisation can also be evaluated with this simple bedside study.

Keywords: Agitated saline, Intracardiac shunt

\section{Background}

Agitated saline contrast echocardiogram (ASC) is an echocardiographic technique which uses agitated saline to primarily diagnose certain disorders like right to left shunt and even various extracardiac shunt physiology. Blood flow mapping with the help of microbubbles can provide diagnostic certainty even in the era of color flow mapping on echocardiogram. The main disadvantage with color flow mapping can be with poor echogenicity as compared to bubble study. Not only are these "bubble studies" inexpensive and technically simple, but they can provide valuable information regarding the presence or absence of intracardiac or intrapulmonary shunts. This can help in diagnosing various clinical syndromes that have evasive etiology such as right ventricular dilatation or dysfunction, platypnea - orthodeoxia syndrome, refractory hypoxemia, and cryptogenic stroke. ASC is well established for detection of patent foramen ovale ( $\mathrm{PFO}$ ) and various other clinical entities if performed and interpreted appropriately. The use of ASC was first described in 1968 in combination with M- mode imaging in six patients referred for ascending aortography ${ }^{1}$.

\section{Physiological Basis and Performing technique}

Preferably a large bore IV cannula is placed in the left arm considering the high incidence of persistent left superior vena cava (SVC) especially with structurally abnormal hearts. 5-10 $\mathrm{ml}$ of $0.9 \%$ saline is mixed with $0.2-1 \mathrm{ml}$ of air and agitated using two different $10 \mathrm{ml}$ syringes connected via a three - way stopcock. This agitated saline is injected rapidly and the echocardiogram machine is set to record at least 20 cardiac cycles .

The ultrasound waves signals from the blood mixed with agitated microbubbles is much intense and echogenic to produce with increased contrast delineation. These microbubbles are too large to cross the micro capillaries of the pulmonary circulation and hence they do not appear in the left side of the heart unless there is right to left shunting present (negative contrast study). The presence of ASC in the left heart structures is consistent with a intracardiac or an intrapulmonary shunt. Current guidelines suggest that the appearance of microbubbles within three to six cardiac cycles after right atrial opacification is considered positive for the presence of an intracardiac shunt, such as a $\mathrm{PFO}^{2}$. The return of bubble contrast into the left side is proportionate to the size of the right-to-left shunt. Based on the opacification, saline contrast echocardiogram can be graded as grade 0 - No bubbles; grade 1 , occasional filling with $<20$ 
bubbles; grade 2, > 20 bubbles, but incomplete opacification; and grade 3-complete intense opacification ${ }^{3-5}$.

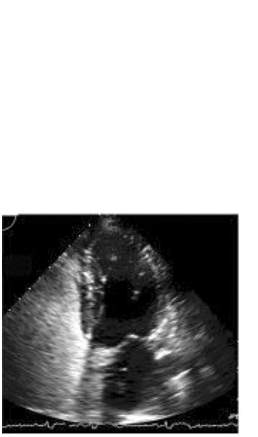

Grade 1 (sma/l)

\begin{tabular}{|l|c|}
\hline Grade & Bubbles* \\
\hline 0 (none) & 0 \\
\hline 1 (small) & $1-9$ \\
\hline 2 (moderate) & $10-30$ \\
\hline 3 (large) & $>30$ \\
\hline
\end{tabular}

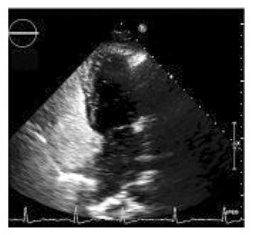

Grade 2 (moderate)



Grade 3 (large)
Figure 1: Grading System for quantification of right to left shunt ${ }^{3}$

In addition, certain physiological maneuvers may be used to provoke right to left shunting like Valsalva maneuver, deep inspiration, cough, or abdominal compression. Generally, the right atrial pressure is always lower than the left atrial pressure so at rest small PFO or intracardiac shunt maybe missed when performing the test at resting condition. These maneuvers may help to increase the sensitivity of detection of PFO compared to doing the test at rest alone.

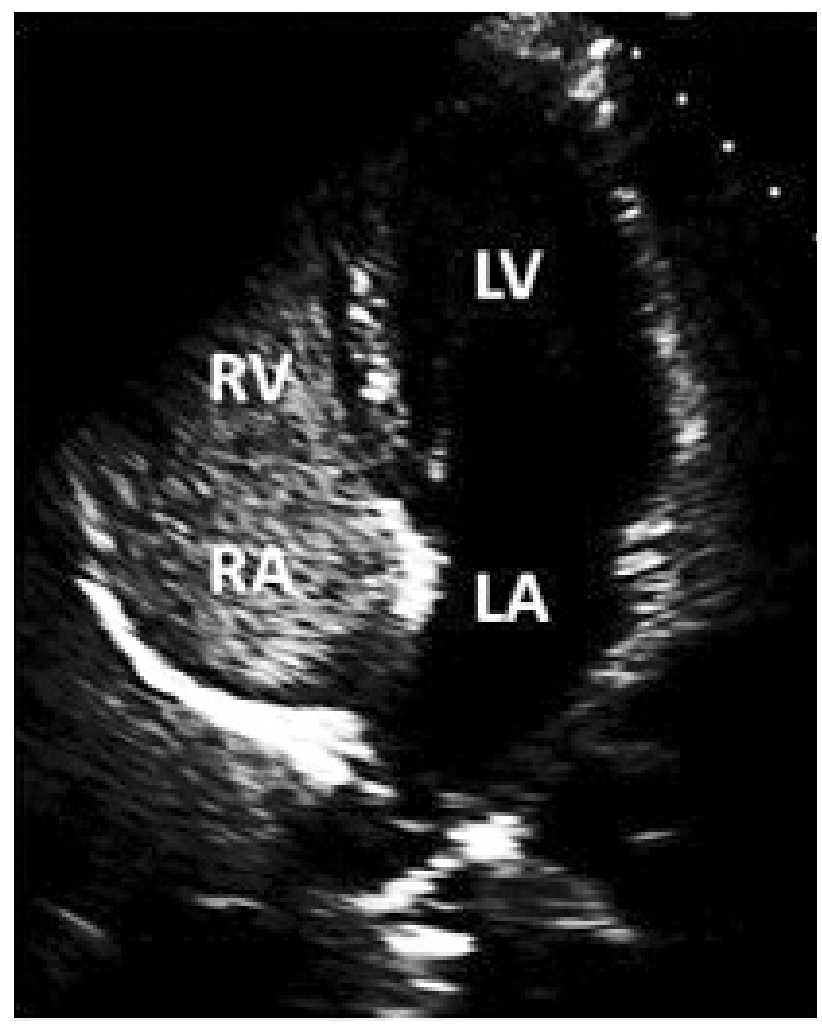

Figure 2: Normal saline contrast echo showing bubbles confined to right sided chambers.

\section{Clinical scenarios where SCE is helpful}

1. Exclude right to left shunting for systemic desaturation

2. Detect intracardiac shunt - Left to right and right to left shunt physiology

Left to right shunt - False echo dropout maybe seen during routine four chamber echocardiogram, or even in poor echo window the correct and precise diagnosis of atrial septal defect may be difficult which can be confirmed with ASC. In these situations, ASC can easily help to confirm the presence of ASD by demonstrating an area of bubble free space (negative contrast shadow) within the Right atrium close to the septum due to the blood flowing from left to right.

\section{Right to left shunt:}

a. Persistent left superior venacava (PLSVC) draining to LA (left atrium) direct or via deficient coronary sinus can be diagnosed with the SCE study. PLSVC is seen in about 0.5 $\%$ population. Microbubbles first appear in LA after left arm injection in case of PLSVC to LA.

b. Right SVC to LA

c. Congenital anomalous total systemic venous drainage Very rare (SVC, IVC, Coronary sinus all connected to LA)

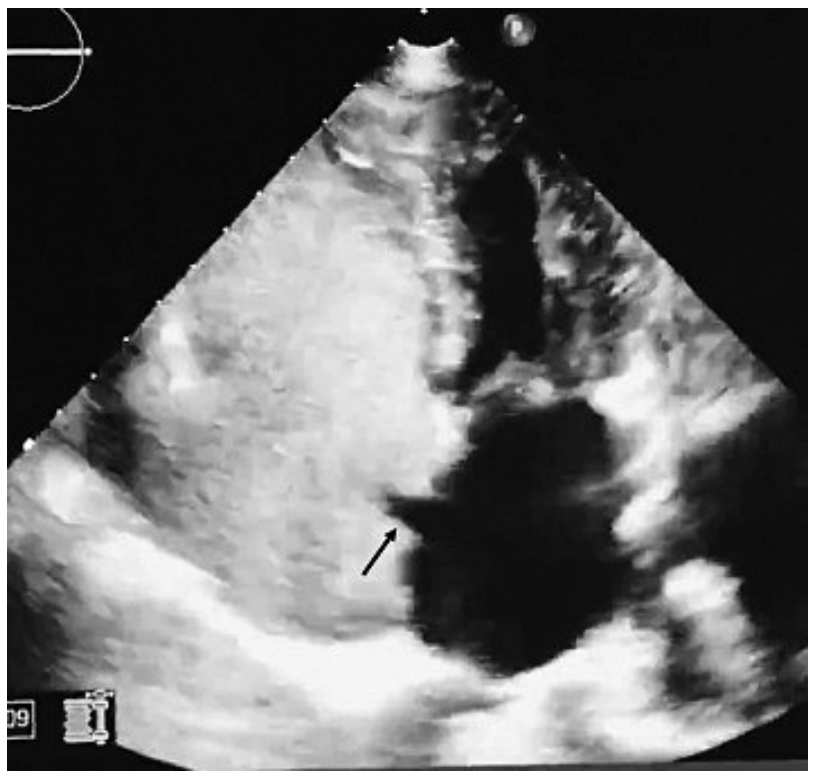

Figure 3: Arrow showing negative contrast effect due to left to right shunt (ASD)

3. Reversal of left to right shunt (Eisenmengerisation): The evaluation of left to right shunt maybe troublesome especially with borderline shunt lesion with very high pulmonary vascular resistance ( PVR) associated with aorto-pulmonary window or large PDA. These undetected primary shunt physiologies maybe missed and wrongly referred to as primary pulmonary hypertension.

4. Right to left shunt in ASD with normal PA pressure: This may occur even in absence of raised pulmonary pressure like - Prominent Eustachian valve, straddling IVC or SVC with sinus venosus ASD, Reduced RV compliance in case of RV myocardial infarction or RV cardiomyopathy etc.

5. Platypnoea-orthodeoxia syndrome: The prevalence of PFO among patients with a cryptogenic stroke has been reported as high as $28-50 \%{ }^{6}$. Cardiac cycles after RA opacification to define PFO separate from extracardiac shunt have also differed among studies mostly using 3-5 cardiac cycles ${ }^{7,8}$.

Dyspnea and hypoxia on upright posture is a common phenomena in patients with significant patent foramen ovale (PFO) . The increase in right to left shunting with sitting from supine position cause desaturation and dyspnea in patients with $\mathrm{PFO}^{9}$. 

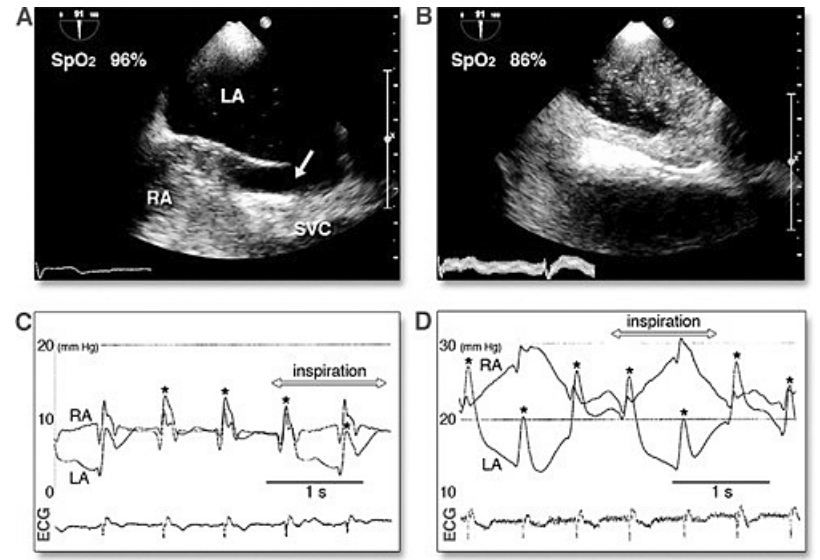

Figure 4: Trans esophageal echo image showing saline entry into left atrium on sitting from supine with simultaneous differential increase in inter-atrial pressure between Right atrium and left atrium . ( Desaturation on sitting - $86 \%$ )

6. Extracardiac (Intrapulmonary shunt): This phenomenon is characterized by delayed appearance of microbubbles in left chambers of the heart while doing SCE; usually after $5-6$ cardiac cycles denoting presence of pulmonary AV malformations (AVM). Diseases associated with pulmonary shunting on saline contrast echocardiography include hereditary hemorrhagic telangiectasia (HHT), hepatopulmonary syndrome, and some congenital heart defects after partial or complete cavopulmonary anastomosis ${ }^{10}$.

\section{Other Uses}

After pericardiocentesis: During localized pericardial collection and drainage catheter in situ, it is of utmost importance to confirm the access into the true pericardial space. ASC maybe used to inject agitated saline to see the microbubbles confined to the pericardial space which confirms the position of needle or the catheter in the right space.

\section{Safety}

Saline contrast echo is a relatively very safe procedure and lifethreatening complications almost never have occurred. Cerebral ischemic events have been reported in $0 \%$ to $0.15 \%$ of patients at the time of or immediately after ASC injection ${ }^{11}$. Air embolism because of injection of agitated saline for SCE is very rare with reported incidence of $0.062 \%$ in a multicenter survey ${ }^{12}$. These events occur almost uniformly in the setting of an intracardiac or intrapulmonary shunt. Symptoms are typically transient or mild, but small strokes may be seen on follow-up MRI. There have been no reported cases of mortality after an ASC study.

\section{Conclusion}

Saline contrast echocardiogram is a relatively cheap and easily available echo technology which can help the clinician in making a wide variety of diagnosis ranging from left to right intracardiac shunts to evaluation of patients with unexplained desaturation. Despite the availability of modern echo machines with high end resolution and better color doppler echocardiogram, bubble study echocardiogram still stands very helpful in various clinical dilemma.

\section{Conflict of Interest \\ None}

\section{Acknowledgement}

None

\section{References}

1. Gramiak R, Shah PM. Echocardiography of the aortic root. Invest Radiol 1968;3:356-66.

https://doi.org/10.1097/00004424-196809000-00011

2. Silvestry FE, CohenMS, ArmsbyLB, BurkuleNJ, FleishmanCE, HijaziZM, et al. Guidelines for the echocardiographic assessment of atrial septal defect and patent foramen ovale: from the American Society of Echocardiography and Society for Cardiac Angiography and Interventions. J Am Soc Echocardiogr 2015;28:910-58 https://doi.org/10.1016/j. echo.2015.05.015

3. Gazzanniga P, Buscarini E, Leandro G, et al: Contrast echocardiohgraphy for pulmonary arteriovenous malfor- mations screening: Does any bubble matter ? Eur J Echocardiogr 2009;10:513-518 https://doi.org/10.1093/ejechocard/ jen317

4. Porter TR, Abdelmoneim S, Belcik JT, McCulloch ML, Mulvagh SL, Olson JJ, Porcelli C, Tsutsui JM, Wei K. Guidelines for the cardiac sonographer in the performance of contrast echocardiography: a focused update from the American Society of Echocardiography. Journal of the American Society of Echocardiography. 2014 Aug 1;27(8):797-810. https://doi. org/10.1016/j.echo.2014.05.011

5. Gupta SK, Shetkar SS, Ramakrishnan S, Kothari SS. Saline contrast echocardiography in the era of multimodality imaging - importance of "bubbling it right". Echocardiography.2015Nov;32(11):170719. https://doi.org/10.1016/j. echo.2014.05.011

6. Handke M, Harloff A, Olschewski M, et al Patent foramen ovale and cryptogenic stroke in older patients. N Engl J Med 2007;357:2262-8. https://doi.org/10.1016/j.echo.2014.05.011

7. Marriott K, Manins V, Forshaw A, et alDetection of right-toleft atrial communication using agitated saline contrast imaging: experience with 1162 patients and recommendations for echocardiography. J Am Soc Echocardiogr 2013;26:96-102. https://doi.org/10.1111/echo.13035

8. Thanigaraj S, Valika A, Zajarias A, et al . Comparison of transthoracic versus transesophageal echocardiography for detection of right-to-left atrial shunting using agitated saline contrast. Am J Cardiol 2005;96:1007-10. https://doi. org/10.1016/j.echo.2012.09.007

9. Shiraishi Y, Hakuno D, Isoda K, Miyazaki K, Adachi T. Platypnea-orthodeoxia syndrome due to PFO and aortic dilation. JACC: Cardiovascular Imaging. 2012 May;5(5):570-1. https://doi.org/10.1016/j.echo.2012.09.007

10. Velthuis S, Buscarini E, Gossage JR, Snijder RJ, Mager JJ, Post MC. Clinical implications of pulmonary shunting on saline contrast echocardiography. Journal of the American Society of Echocardiography. 2015 Mar 1;28(3):255-63. https:// doi.org/10.1016/j.echo.2012.09.007

11. MarriottK, ManinsV, ForshawA, WrightJ, PascoeR.Detectionofright-to- left atrial communication using agitated saline contrast imaging: experi- ence with 1162 patients and recommendations for echocardiography. J Am Soc Echocardiogr 2013; 26:96-102. https://doi.org/10.1016/j.echo.2012.09.007

12. Bommer WJ, Shah PM, Allen H, Meltzer R, Kisslo J. The safety of contrast echocardiography: report of the committee on contrast echocardiography for the American Society of Echocardiography. J Am Coll Cardiol 1984;3:6-13. https:// doi.org/10.1016/j.echo.2012.09.007 